\title{
On the Stability of the Defocusing Mass-Critical Nonlinear Schrödinger Equation
}

\author{
Guangqing Zhang \\ College of Science, Hohai University, Nanjing, China \\ Email: zhanggq@hhu.edu.cn
}

How to cite this paper: Zhang, G.Q. (2016) On the Stability of the Defocusing MassCritical Nonlinear Schrödinger Equation. International Journal of Modern Nonlinear Theory and Application, 5, 115-121. http://dx.doi.org/10.4236/ijmnta.2016.53012

Received: June 27, 2016

Accepted: September 8, 2016

Published: September 16, 2016

Copyright $\odot 2016$ by author and Scientific Research Publishing Inc. This work is licensed under the Creative Commons Attribution International License (CC BY 4.0).

http://creativecommons.org/licenses/by/4.0/

\begin{abstract}
We consider the defocusing mass-critical nonlinear Schrödinger equation in the exterior domain $\Omega$ in $\mathbb{R}^{d}(d \geq 2)$. By analyzing Strichartz estimate and utilizing the inductive hypothesis method, we prove the stability for all initial data in $L^{2}(\Omega)$.
\end{abstract}

\section{Keywords}

Mass-Critical, Stability, Nonlinear Schrödinger Equation

\section{Introduction}

In this short note, we consider the defocusing mass-critical nonlinear Schrödinger equation in the exterior domain $\Omega$ in $\mathbb{R}^{d}(d \geq 2)$ with Dirichlet boundary conditions:

$$
\left\{\begin{array}{l}
i u_{t}+\Delta u=|u|^{\frac{4}{d}} u, \\
u(0, x)=u_{0}(x) \in L^{2}(\Omega), \\
\left.u(t, x)\right|_{x \in \partial \Omega}=0 .
\end{array}\right.
$$

Here $u: \mathbb{R} \times \Omega \rightarrow \mathbb{C}$ and the initial data $u_{0}(x)$ will only be required to the $L^{2}$ space.

This equation has Hamiltonian

$$
M(u(t))=\int_{\mathbb{R}^{d}}|u(t, x)|^{2} \mathrm{~d} x .
$$

As (2) is preserved by (1), we shall refer to it as the mass and often write $M(u)$ or $M$ for $M(u(t))$.

H. Brezis and T. Gallouet [1] considered that $i u_{t}-\Delta u=-k|u|^{2} u$ in $\Omega \times[0, \infty)$, $k \in \mathbb{R}$, the nonlinear Schrödinger equation in $\Omega$ of a bounded domain or an exterior domain of $\mathbb{R}^{2}$ with Dirichlet boundary conditions. In [2], N. Burq, P. Gérard and N. 
Tzvetkov described nonlinear Schrödinger equations in exterior domains. In [3] [4], R. Killip, M. Visan and X. Zhang considered the defocusing energy-critical nonlinear Schrödinger equation and the focusing cubic nonlinear Schrödinger equation in the exterior domain $\Omega$ of a smooth, compact, strictly convex obstacle in $\mathbb{R}^{3}$ with Dirichlet boundary conditions, respectively.

In [5], T. Tao and M. Visan established stability of energy-critical nonlinear Schrödinger equations in $\mathbb{R}^{d}(d \geq 3)$. However, we established stability of mass-critical nonlinear Schrödinger equations in the exterior domain $\Omega$ in $\mathbb{R}^{d}(d \geq 2)$.

Throughout this paper, we restrict ourselves to the following notion of solution.

Definition 1 (solution). Let $I$ be a time interval containing zero, a function $u: I \times \Omega \rightarrow \mathbb{C}$ is called a solution to (1) if it lies in the class $C_{t}^{0} L_{x}^{2}\left(I^{\prime} \times \Omega\right)$ for any compact interval $I^{\prime} \subset I$, and it satisfies the Duhamel formula

$$
u(t)=e^{i t \Delta} u_{0}+i \int_{0}^{t} e^{i(t-s) \Delta}\left(|u|^{\frac{4}{d}} u\right)(s) \mathrm{d} s
$$

for all $t \in I$. The interval $I$ is said to be maximal if the solution cannot be extended beyond $I$. We say $u$ is a global solution if $I=\mathbb{R}$.

In this formulation, the Dirichlet boundary condition is enforced through the appearance of the linear propagator associated to the Dirichlet Laplacian.

Our stability theorem concerns mass-critical stability in $L^{2}(\Omega)$ for the initial-value problem associated to the Equation (1).

Theorem 2 (Stability theorem). Suppose $d \geq 2, I$ is a compact interval and let $\tilde{u}$ be an approximate solution to

$$
\left\{\begin{array}{l}
i \partial_{t} u+\Delta u=|u|^{\frac{4}{d}} u \\
\left.u\right|_{\partial \Omega}=0
\end{array}\right.
$$

in the sense that

$$
i \partial_{t} \tilde{u}+\Delta u=|\tilde{u}|^{\frac{4}{d}} \tilde{u}+e
$$

for some function $e$.

Assume that

$$
\begin{aligned}
& \|\tilde{u}\|_{L_{t}^{\infty} L_{x}^{2}(I \times \Omega)} \leq M \\
& \|\tilde{u}\|_{L_{t, x}^{2(d+2)}} \leq(I \times \Omega) \\
& \frac{2(d)}{d}
\end{aligned}
$$

for some positive constants $M$ and $L$.

Let $t_{0} \in I$ and $u\left(t_{0}\right)$ obey

$$
\left\|u\left(t_{0}\right)-\tilde{u}\left(t_{0}\right)\right\|_{L_{x}^{2}} \leq M^{\prime}
$$

for some $M^{\prime}>0$. Moreover, assume the smallness conditions

$$
\begin{gathered}
\left.\| e^{i\left(t-t_{0}\right) \Delta}\left(u\left(t_{0}\right)-\tilde{u}\left(t_{0}\right)\right)\right) \|_{L_{t, x^{d}}(d+2)}(I \times \Omega) \\
\|e\|_{N^{0}(I)} \leq \epsilon
\end{gathered}
$$


for some $0<\epsilon<\epsilon_{1}$, where $\epsilon_{1}=\epsilon_{1}\left(M, M^{\prime}, L\right)>0$ is a small constant.

Then, there exists a solution $u$ to

$$
\left\{\begin{array}{l}
i \partial_{t} u+\Delta u=|u|^{\frac{4}{d}} u \\
\left.u\right|_{I \times \partial \Omega}=0
\end{array}\right.
$$

on $I \times \Omega$ with initial data $u\left(t_{0}\right)$ at time $t=t_{0}$ satisfying

$$
\begin{gathered}
\|u-\tilde{u}\|_{L_{t, x} \frac{2(d+2)}{d(I \times \Omega)}} \leq C\left(M, M^{\prime}, L\right) \epsilon \\
\|u-\tilde{u}\|_{S^{0}(I)} \leq C\left(M, M^{\prime}, L\right) M^{\prime} \\
\|u\|_{S^{0}(I)} \leq C\left(M, M^{\prime}, L\right) .
\end{gathered}
$$

The rest of the paper is organized as follows. In Section 2, we introduce our notations and state some previous results. In Section 3, we finally prove Theorem 2, except for proving a lemma about approximate solutions.

\section{Preliminaries and Notations}

In this section we summarize some our notations and collect some lemmas that are used in the rest of the paper.

We write $A \lesssim B$ to signify that there is a constant $C>0$ such that $A \leq C B$. We use the notation $A \sim B$ whenever $A \lesssim B \lesssim A$. If the constant $C$ involved has some explicit dependency, we emphasize it by a subscript. Thus $A \lesssim_{u} B$ means that $A \leq C(u) B$ for some constant $C(u)$ depending on $u$. We write $F(z)=z|z|^{\frac{4}{d}}$ for the nonlinearity in (1).

We define that for some $\delta>0$,

$$
\begin{gathered}
\|u\|_{S^{0}(I \times \Omega)}=\sup _{\substack{(q, r) \text { admissible } \\
q \geq 2+\delta}}\|u\|_{L_{t}^{q} L_{x}^{r}(I \times \Omega)}, \\
S^{0}(I \times \Omega)=\left\{u \in C_{t}^{0} L_{x}^{2}(I \times \Omega):\|u\|_{S^{0}(I \times \Omega)}<\infty\right\} .
\end{gathered}
$$

We also define $N^{0}(I \times \Omega)$ to be the space dual to $S^{0}(I \times \Omega)$ with appropriate norm.

With these notations, the Strichartz estimates read as follows:

Theorem 3 (Strichartz estimates [3] [6]). Let $I \subset \mathbb{R}$ be a time interval and let $t_{0} \in I$, then the solution $u: I \times \Omega \rightarrow \mathbb{C}$ to

$$
i u_{t}+\Delta_{\Omega} u=f
$$

satisfies

$$
\|u\|_{S^{0}} \leq\left\|u\left(t_{0}\right)\right\|_{L^{2}(\Omega)}+\|f\|_{N^{0}(I)} .
$$

Proposition 4 (Local well-posedness). Given $E>0$, there exists $\delta_{0}=\delta_{0}(E)>0$ such that if $\|\phi\|_{L^{2}(\Omega)} \leq E$ and

$$
\left\|e^{i t \Delta} \phi\right\|_{L_{t}^{q} L_{x}^{r}(I \times \Omega)} \leq \delta_{0}
$$

on some interval $I \ni 0,|I| \leq 1$, then there exists a unique solution $u \in C_{t}^{0} L_{x}^{2}(I \times \Omega)$ 
of (1) satisfying $u(0)=\phi$. Besides,

$$
\left\|u-e_{S^{0}(I \times \Omega)}^{i t \Delta}\right\| \lesssim_{E}\left\|e^{i t \Delta} \phi\right\|_{L_{t}^{q} L_{x}^{r}(I \times \Omega)}^{\frac{3}{2}} .
$$

The quantities $M(u)$ defined in (2) are conserved on $I$.

\section{Proof of Theorem 2}

We need the following lemma to prove this theorem.

Lemma 1. Let I be a compact interval and let $\tilde{u}$ be an approximate solution to

$$
\left\{\begin{array}{l}
i \partial_{t} u+\Delta u=|u|^{\frac{4}{d}} u \\
\left.u\right|_{I \times \partial \Omega}=0
\end{array}\right.
$$

in the sense that

$$
i \partial_{t} \tilde{u}+\Delta \tilde{u}=|\tilde{u}|^{\frac{4}{d}} \tilde{u}+e
$$

for some function $e$.

Assume that

$$
\|\tilde{u}\|_{L_{t}^{\infty} L_{x}^{2}(I \times \Omega)} \leq M
$$

for some positive constant $M$.

Let $t_{0} \in I$ and $u\left(t_{0}\right)$ be such that

$$
\left\|u\left(t_{0}\right)-\tilde{u}\left(t_{0}\right)\right\|_{L^{2}} \leq M^{\prime}
$$

for some $M^{\prime}>0$.

Assume also the smallness conditions

$$
\begin{gathered}
\|\tilde{u}\|_{L_{t, x}}^{\frac{2(d+2)}{d}(I \times \Omega)} \leq \epsilon_{0} \\
\left\|e^{i\left(t-t_{0}\right) \Delta}\left(u\left(t_{0}\right)-\tilde{u}\left(t_{0}\right)\right)\right\|_{L_{t, x^{x}}\left(\frac{2(d+2)}{(I \times \Omega)}\right.} \leq \epsilon \\
\|e\|_{N^{0}(I)} \leq \epsilon
\end{gathered}
$$

for some $0<\epsilon \leq \epsilon_{0}$, where $\epsilon_{0}=\epsilon_{0}\left(M, M^{\prime}\right)>0$ is a small constant.

Then, there exists a solution $u$ to

$$
\left\{\begin{array}{l}
i \partial_{t} u+\Delta u=|u|^{\frac{4}{d}} u \\
\left.u\right|_{I \times \partial \Omega}=0
\end{array}\right.
$$

on $I \times \Omega$ with initial data $u\left(t_{0}\right)$ at $t=t_{0}$ satisfying

$$
\begin{gathered}
\|u-\tilde{u}\|_{L_{t, x^{d}}}^{\frac{2(d+2)}{(I \times \Omega)}} \lesssim \epsilon \\
\|u-\tilde{u}\|_{S^{0}(I)} \lesssim M^{\prime} \\
\|u\|_{S^{0}(I)} \lesssim M+M^{\prime} \\
\left\|\left.\left|u^{\frac{4}{d}} u-\right| \tilde{u}\right|^{\frac{4}{d}} \tilde{u}\right\|_{N^{0}(I)} \lesssim \epsilon .
\end{gathered}
$$


Proof of Lemma 1. By symmetry, we may assume $t_{0}=\inf I$. Let $w=u-\tilde{u}$, then $w$ satisfies the following problem

$$
\left\{\begin{array}{l}
i \partial_{t} w+\Delta w=F(\tilde{u}+w)-F(\tilde{u})-e \\
w\left(t_{0}\right)=u\left(t_{0}\right)-\tilde{u}\left(t_{0}\right) \\
\left.w\right|_{I \times \partial \Omega}=0
\end{array}\right.
$$

where $F(u)=|u|^{\frac{4}{d}} u$.

For $t \in I$, we define

$$
A(t)=\|F(\tilde{u}+w)-F(\tilde{u})\|_{N^{0}\left(\left[t_{0}, t\right]\right)} .
$$

By (19),

$$
\begin{aligned}
& A(t) \lesssim\|F(\tilde{u}+w)-F(\tilde{u})\|_{L_{t, x} \frac{2(d+2)}{d+4}}\left(\left[t_{0}, t\right] \times \Omega\right) \\
& \lesssim\left\||\tilde{u}+w|^{\frac{4}{d}}(\tilde{u}+w)-|\tilde{u}|^{\frac{4}{d}} \tilde{u}\right\|_{L_{t, x} \frac{2(d+2)}{d+4}\left(\left[t_{0}, t\right] \times \Omega\right)} \\
& \lesssim\left\|\left(|\tilde{u}|^{\frac{4}{d}}+|w|^{\frac{4}{d}}\right) w\right\|_{{\frac{2(d+2)}{L_{t, x}+4}}_{\left(\left[t_{0}, t\right] \times \Omega\right)}}
\end{aligned}
$$

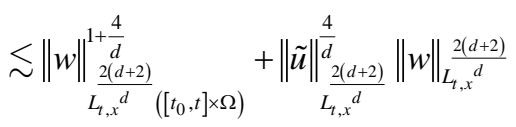

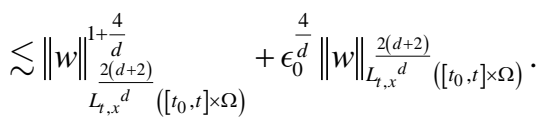

On the other hand, by Strichartz, (20), (21), we get

$$
\begin{aligned}
& \|w\|_{L_{t, x}}^{\frac{2(d+2)}{d}}\left(\left[t_{0}, t\right] \times \Omega\right) \\
& \lesssim\left\|e^{i\left(t-t_{0}\right) \Delta} w\left(t_{0}\right)\right\| \frac{2(d+2)}{L_{t, x} d}\left(\left[t_{0}, t\right] \times \Omega\right) \\
& \lesssim A(t)+\epsilon
\end{aligned}
$$

Combining (27) and (28), we obtain

$$
A(t) \lesssim(A(t)+\epsilon)^{1+\frac{4}{d}}+\epsilon_{0}^{\frac{4}{d}}(A(t)+\epsilon)+\epsilon .
$$

By bootstrapping, we see if $\epsilon_{0}$ is taken sufficiently small,

$$
A(t) \lesssim \epsilon \text { for } t \in I \text {, }
$$

which implies (26).

Using (26) and (28), we see (23).

Moreover, by Strichartz, (18), (21) and (26),

$$
\begin{aligned}
\|w\|_{S^{0}(I)} & \lesssim\left\|w\left(t_{0}\right)\right\|_{L_{x}^{2}}+\|F(\tilde{u}+w)-F(\tilde{u})\|_{N^{0}(I)}+\|e\|_{N^{0}(I)} \\
& \lesssim M^{\prime}+\epsilon,
\end{aligned}
$$

which establishes (24) for $\epsilon_{0}=\epsilon_{0}\left(M^{\prime}\right)$ sufficiently small.

To show (25), we use Strichartz, (17), (18), (26), (19), 


$$
\begin{aligned}
\|u\|_{S^{0}(I)} & \lesssim\left\|u\left(t_{0}\right)\right\|_{L_{x}^{2}}+\|F(u)\|_{N^{0}(I)} \\
& \lesssim\left\|\tilde{u}\left(t_{0}\right)\right\|_{L^{2}}+\left\|u\left(t_{0}\right)-\tilde{u}\left(t_{0}\right)\right\|_{L^{2}}+\|F(u)-F(\tilde{u})\|_{N^{0}(I)}+\|F(\tilde{u})\|_{N^{0}(I)} \\
& \lesssim M+M^{\prime}+\epsilon+\|\tilde{u}\|_{\substack{L_{t, x^{d}}+\frac{4}{d}(I \times 2) \\
L_{(I \times \Omega)}}} \lesssim M+M^{\prime}+\epsilon+\epsilon_{0}^{1+\frac{4}{d}} .
\end{aligned}
$$

Choosing $\epsilon_{0}=\epsilon_{0}\left(M, M^{\prime}\right)$ sufficiently small, this finishes the proof of the lemma.

We now turn to the proof of stability theorem.

Proof of Theorem 2. We now subdivide $I$ into $J \sim\left(1+\frac{L}{\epsilon_{0}}\right)^{\frac{2(d+2)}{d}}$ subintervals $I_{j}=\left[t_{j}, t_{j+1}\right], \quad 0 \leq j \leq J-1$, such that

$$
\|\tilde{u}\|_{L_{t, x}}^{\frac{2(d+2)}{d}\left(I_{j} \times \Omega\right)} \leq \epsilon_{0},
$$

where $\epsilon_{0}=\epsilon_{0}\left(M, 2 M^{\prime}\right)$ as in the lemma.

We need to replace $M^{\prime}$ by $2 M^{\prime}$ as the mass of the difference $u-\tilde{u}$ might grow slightly in time.

By choosing $\epsilon_{1}$ sufficiently small depending on $J, M$ and $M^{\prime}$, we can apply the lemma to obtain for each $j$ and all $0<\epsilon<\epsilon_{1}$,

$$
\begin{aligned}
& \|u-\tilde{u}\|_{L_{t, x^{d}}^{d}}^{\frac{2(d+2)}{\left(I_{j} \times \Omega\right)}} \leq C(j) \epsilon \\
& \|u-\tilde{u}\|_{S^{0}\left(I_{j}\right)} \leq C(j) M^{\prime} \\
& \|u\|_{S^{0}\left(I_{j}\right)} \leq C(j)\left(M+M^{\prime}\right) \\
& \|F(u)-F(\tilde{u})\|_{N^{0}\left(I_{j}\right)} \leq C(j) \epsilon,
\end{aligned}
$$

provided we can show that analogues of (8) and (9) hold with $t_{0}$ replaced by $t_{j}$.

In order to verify this, we use an inductive argument.

By Strichartz, (8), (10) and the inductive hypothesis,

$$
\begin{aligned}
& \left\|u\left(t_{j}\right)-\tilde{u}\left(t_{j}\right)\right\|_{L_{x}^{2}} \\
& \lesssim\left\|u\left(t_{0}\right)-\tilde{u}\left(t_{0}\right)\right\|_{L_{x}^{2}}+\|F(u)-F(\tilde{u})\|_{N^{0}\left(\left[t_{0}, t_{j}\right]\right)}+\|e\|_{N^{0}\left(\left[t_{0}, t_{j}\right]\right)} \\
& \lesssim M^{\prime}+\sum_{k=0}^{j-1} C(k) \epsilon+\epsilon .
\end{aligned}
$$

Similarly, by Strichartz, (9), (10) and the inductive hypothesis, we see

$$
\begin{aligned}
& u\left(t_{0}\right)-\tilde{u}\left(t_{0}\right)=e^{i\left(t_{0}-t_{j}\right) \Delta}\left(u\left(t_{j}\right)-\tilde{u}\left(t_{j}\right)\right)+\int_{t_{j}}^{t_{0}} e^{i\left(t_{0}-\tau\right) \Delta}(F(u)-F(\tilde{u})-e)(\tau) \mathrm{d} \tau \\
& \Rightarrow e^{i\left(t-t_{0}\right) \Delta}\left(u\left(t_{0}\right)-\tilde{u}\left(t_{0}\right)\right)=e^{i\left(t\left(t t_{j}\right) \Delta\right.}\left(u\left(t_{j}\right)-\tilde{u}\left(t_{j}\right)\right)+\int_{t_{j}}^{t_{0}} e^{i(t-\tau) \Delta}(F(u)-F(\tilde{u})-e)(\tau) \mathrm{d} \tau,
\end{aligned}
$$

so we see

$$
\begin{aligned}
& \left\|e^{i\left(t-t_{j}\right) \Delta}\left(u\left(t_{j}\right)-\tilde{u}\left(t_{j}\right)\right)\right\|_{L_{t, x^{d}}} \frac{2(d+2)}{\left.I_{j} \times \Omega\right)} \\
& \lesssim\left\|e^{i\left(t-t_{0}\right) \Delta}\left(u\left(t_{0}\right)-\tilde{u}\left(t_{0}\right)\right)\right\|_{L_{L_{,}, d^{x}}\left(d_{\left(I_{j} \times \Omega\right)} \times \Omega\right)}+\|e\|_{N^{0}\left(\left[\left[_{0}, t_{j}\right]\right)\right.}+\|F(u)-F(\tilde{u})\|_{N^{0}\left(\left[t_{0}, t_{j}\right]\right)} \lesssim \epsilon+\sum_{k=0}^{j-1} C(k) \epsilon .
\end{aligned}
$$


Choosing $\epsilon_{1}$ sufficiently small depending on $J, M$ and $M^{\prime}$, we can guarantee that the hypotheses of the lemma continue to hold as $j$ varies.

\section{Conclusion}

In this paper, we consider a mass-critical stability of the defocusing mass-critical nonlinear Schrödinger equation. Then we prove two different types of perturbation to show the stability of nonlinear Schrödinger equation.

\section{Acknowledgements}

The research of Guangqing Zhang has been partially supported by the NSF grant of China (No. 51509073) and also “The Fundamental Research Funds for the Central Universities" (No. 2014B14214). The author would like to thank his tutor Zhen Hu for helpful conversations. The author also thanks the referees for their time and comments.

\section{References}

[1] Brezis, H. and Gallouet, T. (1980) Nonlinear Schrödinger Evolution Equations. Nonlinear Analysis, 4, 677-681.

[2] Burq, N., Gérard, P. and Tzvetkov, N. (2004) On Nonlinear Schrödinger Equations in Exterior Domains. Annales de P Institut Henri Poincaré (C) Analyse Non Linear Analysis, 21, 295-318.

[3] Killip, R., Visan, M. and Zhang, X. (2012) Quintic NLS in the Exterior of a Strictly Convex Obstacle. Mathematics. arXiv:1208.4904.

[4] Killip, R., Visan, M. and Zhang, X. (2015) The Focusing Cubic NLS on Exterior Domains in Three Dimensions. Mathematics, 89, 335-354.

[5] Tao, T. and Visan, M. (2005) Stability of Energy-Critical Nonlinear Schrödinger Equations in High Dimensions. Electronic Journal of Differential Equations, 2, 357-370.

[6] Keel, M. and Tao, T. (1997) Endpoint Strichartz Estimates. American Journal of Mathematics, 120, 955-980.

Submit or recommend next manuscript to SCIRP and we will provide best service for you:

Accepting pre-submission inquiries through Email, Facebook, LinkedIn, Twitter, etc. A wide selection of journals (inclusive of 9 subjects, more than 200 journals)

Providing 24-hour high-quality service

User-friendly online submission system

Fair and swift peer-review system

Efficient typesetting and proofreading procedure

Display of the result of downloads and visits, as well as the number of cited articles

Maximum dissemination of your research work

Submit your manuscript at: http://papersubmission.scirp.org/ 